\title{
The role of axes of elongation and symmetry in rotated object naming
}

\author{
MARY-ELLEN LARGE, PATRICIA A. MCMULLEN, and JEFF P. HAMM \\ Dalhousie University, Halifax, Nova Scotia, Canada
}

\begin{abstract}
Many theorists have postulated that axes of elongation and/or symmetry play an important role in the recognition of objects. In this paper, evidence is presented that mitigates this claim from independent assessments of the effects of axes of elongation or symmetry on the time to name rotated line drawings of common objects. This conclusion was further supported in a stronger test in which both of these variables were orthogonally controlled, the aspect ratio of elongation was manipulated, and only objects that were completely geometrically symmetrical or asymmetrical were used. In all the experiments, objects were named for several blocks to determine the influence of these variables on effects of orientation with practice. Symmetry was found to diminish the effects of orientation after practice in naming the object set, and the effects of the most extreme orientation tested $\left(120^{\circ}\right.$ from upright $)$ were diminished when both axes defined the same orientation, relative to when they defined different orientations. Contrary to many theories, these findings relegate the axes of symmetry and elongation to relatively minor roles during object identification.
\end{abstract}

An important characteristic of the visual system is the ease with which familiar shapes are recognized despite differences in the retinal image owing to circumstances particular to each instance of object viewing. A number of theorists (Biederman, 1987; Marr, 1982; Marr \& Nishihara, 1978) have argued that the visual system constructs representations of objects from the viewpoint of the observer. For recognition to proceed, these view-centered representations are then matched to object-centered representations stored in memory. According to Marr, both view-centered and object-centered representations need a spatial coordinate system to code for the relations between object parts. A transformation process is also necessary to convert from a viewer-centered coordinate system to an object-centered coordinate system. Marr reasoned that the principal axes of objects can act as the origin for these coordinate systems, since they are geometrically stable, and he advocated that these principal axes play a primary role in the transformation process. This study focused on the possible role the axes of elongation and/or symmetry may play in object recognition by observing their effects on naming rotated line drawings of common objects.

In a number of recent studies, the perceptual importance of the axes of symmetry and elongation has been investigated. Quinlan and Humphreys (1993) performed

This work was supported by Natural Sciences and Engineering Research Council of Canada and Human Frontiers Science Program awards to the second author. We acknowledge the assistance of Natasha Wirtanen, Vanessa McCarthy, and David Rousein in data collection and John Christie for his help in setting up the program for running Experiment 3. Correspondence concerning this article should be addressed to M.-E. Large, Department of Psychology, Dalhousie University, Halifax, NS, B3H 4J1 Canada (e-mail: mlarge@ is2.dal.ca). experiments in which they examined the processing of a set of two-dimensional, four-sided geometric shapes in which the intrinsic axes of symmetry and elongation were varied in an orthogonal fashion. In Experiment 1, participants were required to draw a line through the figures that corresponded to the axes that naturally went with the shapes. They found that most participants were likely to draw a line through an axis of symmetry when the shape had one. On the other hand, participants were inconsistent in drawing a line corresponding to the axis of elongation. They concluded that, unlike the axis of symmetry, the axis of elongation was perceptually unimportant.

However, Sekuler (1996; Sekuler \& Swimmer, 2000) argued that Quinlan and Humphreys (1993) did not take into account the relative salience of elongation and symmetry cues. Sekuler modified Palmer's (1990) paradigm, which examined the effects of elongated surrounds on the perception of the orientation of equilateral triangles. She demonstrated that symmetrical and asymmetrical elongated surrounds influenced the derivation of reference frames when the salience of elongation relative to symmetry was manipulated by increasing the aspect ratio of elongated surrounds. More relevant to this study, Sekuler and Swimmer found that axes of elongation internal to the object also influenced major axis judgments. Using simple two-dimensional shapes that varied in aspect ratio and number of symmetrical elements, Sekuler and Swimmer were able to manipulate the salience of symmetry and elongation cues while observers judged the orientation of the primary axis. They found that both symmetry and elongation were sufficient to derive primary axes and that these axes were not isolated in their influence.

The problem with the above studies is that they focused on explicitly defining the primary axes of objects 
and did not address the implicit role these axes might play in object identification. The observers were required to define the primary axis of an object by drawing it in or by responding with a key to indicate whether the primary axis was horizontal or vertical, the logic being that the ability to perceive the primary axes of objects was related to their involvement in object identification. Recently, this effect was shown again. Liu and Cooper (2001) demonstrated that symmetry primed judgments about the symmetry of nonsense objects. However, symmetry did not prime an object decision task, which is likely a closer task to object identification.

Marr's (1982) theory makes strong claims about the role of these principal axes in object identification. However, in previous reports, the role of symmetry in object identification has not been explicitly tested. In order to test his claims, it is necessary to investigate the effects of the axes of elongation and symmetry in a task that involves object recognition and/or identification.

Ling and Sanocki (1995) went some way toward addressing this problem by providing evidence that the axis of elongation may be an important component of object representations by priming the identification of objects. Their primes consisted of the major axes, the actual edges, expanded edges, contracted edges, a four-sided frame that delimited the stimulus location and orientation, and a circle. Of particular interest, they found that the primes containing major axis information facilitated object identification, in comparison with a reference frame prime that marked the outer edges of the object and a circle prime that controlled for attention attraction and location information. Ling and Sanocki concluded that axis information facilitated identification by activating an abstract visual model of the object that contained information about structural relations.

A problem with Ling and Sanocki's (1995) study is that it used only a small number of stimuli. They were all airplanes made up of the same outline, with small differences in the shape and location of their parts. Identification of the airplanes was based on differences in the shape of the windows. Since all that changed between stimuli were the parts, coding for their location was no doubt crucial for discrimination. It may be that the axis of elongation is less useful in the context of identifying many objects whose parts differ by a greater magnitude. Furthermore, their experiment did not address other issues brought up in Marr's (1982) theory, such as the role the axes may play in the transformation from view-centered to object-centered coordinate systems, which he argued was a necessary stage in object identification. The present study extends the investigation of the effects of principal axes on object identification by examining the effects of the axes of elongation and symmetry on the naming of many different rotated objects.

Numerous studies have demonstrated that the time to name line drawings of familiar objects increases linearly with stimulus orientations between $0^{\circ}$ and $120^{\circ}$ (Jolicœur, 1985; McMullen \& Jolicœur, 1990; Murray, 1995). This pattern of naming latencies is consistent with normal- ization (or transformation, in Marr's, 1982, terms) of a view-centered representation of the input image through the shortest angular distance to the upright to find a match with object representations in long-term memory that are aligned with a canonical upright (Jolicœur, 1990; Pinker, 1984). However, this effect of orientation diminishes as the same objects are repeatedly named at different orientations (Jolicœur, 1985). To account for the initial effect of orientation and its diminution with practice, Jolicœur (1990; Jolicœur \& Humphrey, 1998) hypothesized two routes to rotated object naming: one in which the entire rotated image is normalized to the upright before a match is made with long-term object representations and a second in which local, orientation-invariant features are sufficient to make this match (see, also, Humphreys \& Riddoch, 1984, 1985; Riddoch \& Humphreys, 1986). During the first of these processes, global shape is determined as Marr described, by spatially relating object parts to intrinsic frames of reference aligned with an object's main axis or axis of symmetry. This route predominates when objects are first named and is clearly orientation sensitive. The second of these proposed routes predominates when objects have been repeatedly named in different orientations and is less sensitive to orientation.

McMullen and Jolicœur (1992) investigated the effects of orientation on making discriminations about the location of the tops and bottoms of rotated objects. They concluded that processing the spatial relations of object features was crucial in making these discriminations. They also observed that orientation effects for naming and top-bottom discriminations were similar and that previous viewing of objects in the context of top-bottom discriminations resulted in reduced effects of orientation in a subsequent naming task. On this basis, they argued that orientation effects associated with naming rotated objects for the first time are due to processing the location of object features. They postulated that the major internal axis of an object acts as a spatial coordinate referent for locating object parts.

If the orientation normalization route has been correctly characterized by Jolicœur (1990; Jolicœur \& Humphrey, 1998), objects with axes of elongation or symmetry ought to show different effects of orientation when first named, relative to objects without these axes. Indeed, Tarr and Pinker (1990) demonstrated that orientation effects are reduced for two-dimensional figures that have bilateral symmetry. They argued that object-centered representations could encode parts and their spatial relations only along one dimension. Since the parts for symmetrical shapes are repeated about the axis of symmetry, the visual system can access an orientation-invariant object-centered representation. In contrast, with asymmetrical figures, it is necessary to distinguish between the parts and their relations along two dimensions, requiring that they be normalized before identification can occur.

In support of Tarr and Pinker's (1990) findings, McMullen and Farah's (1991) post hoc analysis of naming 
times for rotated real objects found smaller effects of orientation for symmetrical objects than for asymmetrical objects. However, an advantage for symmetrical figures was present only after repeated naming. They concluded that the property of symmetry enables access to objectcentered representations only after experience in recognizing a particular exemplar. Neither of the above studies was designed to distinguish the effects of symmetry when rotated objects are first identified.

In the following three experiments, we examined whether axes of symmetry and elongation would influence an identification task in which more complex realworld objects were used. They were also designed to test whether these axes would have an effect on the normalization of rotated objects on first naming. In each of the experiments, the observers were required to name line drawings of common objects that were presented at multiple orientations. Given evidence that these axes may influence practice effects (McMullen \& Farah, 1991) by diminishing effects of orientation, objects were named for a number of blocks. Objects were grouped according to their axis properties available from a single viewpoint that maximized the salience of either or both axes of symmetry and elongation. Naming times and errors for each group of objects were compared.

It was expected that if axes of elongation and/or symmetry were used in the normalization of rotated images, an interaction between orientation and axis type would be present in the first block of naming, where the presence of either axis or both would reduce the effects of orientation-the assumption being that the presence of an axis would provide orientation information or in some way facilitate encoding of features relative to it, although it should be noted that previous studies in which orientation precues were provided before rotated objects were named failed to influence effects of orientation (Gauthier \& Tarr, 1997; Gibson \& Peterson, 1994; McMullen, Hamm, \& Jolicœur, 1995). If the axes of symmetry and/or elongation underlie practice effects with the naming of rotated objects, it would be expected that, after repeated naming, effects of orientation would diminish at different rates in relation to the axis property. In Experiment 1, effects of the axis of elongation were investigated; in Experiment 2, effects of the axis of symmetry were investigated; and in Experiment 3, the effects of both axes were investigated in a design in which these two variables were controlled orthogonally to each other.

\section{EXPERIMENT 1}

If an axis of elongation is present, it is either aligned with the top-bottom axis or perpendicular to it. This information about the location of the top of the image could constrain the direction of image rotation and result in smaller effects of orientation. Chambers, McBeath, Schiano, and Metz (1999) found that observers reliably classified objects as similar when their tops were matched, but not when their bottoms matched, indicating that tops are perceptually more salient than bottoms. However, when information about the location of the top of rotated objects was available prior to rotated object naming in the form of an orientation precue, no reduction in orientation effects was found (Gauthier \& Tarr, 1997; Gibson \& Peterson, 1994; McMullen et al., 1995). Nevertheless, it is possible that only top-bottom information intrinsic to the object, such as an object axis, can inform about the location of the top and thereby reduce effects of orientation.

Objects with and without a major axis or axis of elongation were named. The top of half of the elongated objects was located at one end of the long axis (tall objects), whereas the top of the other elongated objects was located at one end of the short axis (wide objects). A remaining third of the stimuli were judged to be as wide as they were tall (non-elongated objects). If an axis of elongation can indicate the location of the top of objects and so guide the direction of rotation, objects with an axis should show smaller effects of orientation than do those without. Differences in the effects of orientation between tall and wide objects should help elucidate how these axes are used.

\section{Method}

Participants. Forty-eight undergraduate students participated voluntarily or received credit toward an introductory psychology course. All the participants had normal or corrected-to-normal vision and reported English as their first language.

Stimuli and Apparatus. Presentation of stimuli and recording of reaction times were computer controlled. A KODAK carousel slide projector fitted with an electronic shutter was used to rear project the stimuli for a fixed exposure time onto a mylar screen. The screen was overlaid with a black paper mat, and the image was presented within a hole cut in the mat with a diameter of $5.85^{\circ}$ of visual angle.

Fourteen participants who did not participate in the naming experiment rated 120 objects from Snodgrass and Vanderwart (1980) on the basis of their elongation (tall, wide, or non-elongated; see Appendix A). All the objects had a distinct top and bottom and were used in McMullen and Jolicœur (1992). From these objects, 72 were selected on the basis of their mean elongation ratings $(0=$ non-elongated, $1=$ tall, and $-1=$ wide), such that 24 objects made up the categories of tall, wide, or non-elongated. All the objects were photographed with high-contrast black-and-white slide film and were mounted in slide mounts. Every object was photographed in six orientations: $0^{\circ}, 60^{\circ}, 120^{\circ}, 180^{\circ}, 240^{\circ}$, and $300^{\circ}$ clockwise from upright.

Procedure. Each experimental session consisted of six blocks of 72 trials. Four random orders of slides were created, with the following constraints. Within each block, 24 trials were wide objects, 24 were non-elongated objects, and 24 were tall objects. These three sets of 24 stimuli were further divided by orientation; in each set of 24 stimuli, 6 objects were shown at $0^{\circ}, 6$ objects were shown at $180^{\circ}$, and the remaining 12 objects were divided evenly and shown at orientations of $60^{\circ}, 120^{\circ}, 240^{\circ}$, and $300^{\circ}$, respectively. Naming times for objects shown at $60^{\circ}$ and $300^{\circ}$ and objects shown at $120^{\circ}$ and $240^{\circ}$ were collapsed for analysis, since there was no theoretical reason to assume that rotation clockwise should produce different results than rotation counterclockwise. No object was repeated during a single block. The same 72 stimulus objects were seen at different orientations across the four blocks so that each object was shown once at $0^{\circ}$ and once at $180^{\circ}$. For orientations $60^{\circ}, 120^{\circ}, 240^{\circ}$, and $300^{\circ}$, the trials were split evenly, and the slides were counterbalanced. For instance, half the participants saw a "car" at $60^{\circ}$, and the other half saw it at $300^{\circ}$. This procedure resulted in each subject's seeing six of each object type at each of the four orientations 
(with $60^{\circ}$ and $300^{\circ}$ collapsed and $120^{\circ}$ and $240^{\circ}$ collapsed) within a block. Twelve practice trials were presented with 3 objects at each orientation. Objects named during practice were never presented on experimental trials. Every participant then completed four blocks of experimental trials, with the blocks shown in a Latin square across participants. This ensured that every object was seen an equal number of times in every orientation across participants.

Trials were initiated when the experimenter pressed a computer key. The electronic shutter opened to display an object, and a millisecond timer was initiated. Vocal naming responses tripped a voice key that stopped the timer and closed the electronic shutter. Responses were scored as correct, incorrect, or spoiled. Responses that were considered correct are available in McMullen and Jolicœur (1990). A trial was considered spoiled if the voice key failed to trip or tripped prematurely (i.e., the participant said "Ah..."), if the slide failed to project properly, or if the participant failed to respond within $4,000 \mathrm{msec}$. Spoiled trials were not included in the analyses. Reaction time and all pertinent trial information were stored to disk for later analysis.

\section{Results}

Outliers were eliminated, according to the modified recursive method recommended by Van Selst and Jolicœur (1994), before the analysis of naming times in all three experiments reported in this paper. This resulted in an average of $3 \%$ of the data per subject being eliminated as outliers. The contrast weights used with linear analyses for orientations of $0^{\circ}-180^{\circ}$ were $(-3,-1,1,3)$. The contrast weights used with linear analyses for orientations of $0^{\circ}-120^{\circ}$ were $(-1,0,1)$. Analyses were conducted on orientations between $0^{\circ}$ and $180^{\circ}$ and between $0^{\circ}$ and $120^{\circ}$. The analysis of orientations between $0^{\circ}$ and $120^{\circ}$ was included because many studies of orientation effects in rotated object identification have shown a considerable decrease in naming times at $180^{\circ}$. Jolicœur (1990; Jolicœur \& Humphrey, 1998) postulated that this decrease in response time was due to identification of features that are orientation invariant at $180^{\circ}$ (see Murray, 1997, for an alternative explanation). Accordingly, the process of normalization within the plane, which was the focus of this study, may not be utilized at this orientation.

Mean naming times for all blocks. Two repeated measures analyses of variance (ANOVAs) were performed: one analysis for orientations of $0^{\circ}$ to $180^{\circ}$, with block (4), orientation (4), and object elongation type (3) as within-subject factors, and another with the same design for orientations of $0^{\circ}$ to $120^{\circ}$ with block (4), orientation (3) and object elong ation type (3). For the purpose of clarity, the results from the analysis of orientations between $0^{\circ}$ and $120^{\circ}$ are reported only when they differ in significance from those for orientations $0^{\circ}-180^{\circ}$. There was a main effect of block $\left[F(3,141)=72.81, M S_{\mathrm{e}}=\right.$ $27,585, p<.0001]$, indicating a decrease in naming times as a result of practice. As has often been demonstrated, large effects of orientation were found $[F(3,141)=$ $\left.54.59, M S_{\mathrm{e}}=8,956.7, p<.0001\right]$. Trend analyses revealed both linear $[F(1,47)=91.41, p<.0001]$ and quadratic $[F(1,47)=65.28, p<.0001]$ components to this effect for orientations of $0^{\circ}-180^{\circ}$ and only a linear component for orientations of $0^{\circ}-120^{\circ}[F(1,47)=100.9, p<$ .0001]. An effect of object elongation type was found
$\left[F(2,94)=23.51, M S_{\mathrm{e}}=18,774.7, p<.0001\right]$. Planned contrasts revealed that wide objects $(M=830.28 \mathrm{msec})$ were named more quickly than tall objects $[M=$ $876.68 \mathrm{msec} ; F(1,47)=41.36, p<.0001]$. Interestingly, elongated objects were named as quickly as non-elongated objects $[F(1,47)=3.2, p<.08]$. There was a significant interaction between block and orientation $[F(9,423)=$ $\left.1.878, M S_{\mathrm{e}}=15,967.9, p=.05\right]$, demonstrating a diminished effect of orientation over blocks. Most important for the theory tested here, there was an interaction between orientation and object elongationtype $[F(6,282)=$ 2.15, $\left.M S_{\mathrm{e}}=7,534.28, p=.05\right]$ for orientations of $0^{\circ}-180^{\circ}$ that had a linear component $[F(1,47)=4.31$, $p=.04]$. However, this interaction was not present for orientations of $0^{\circ}-120^{\circ}(F<1)$. This suggests that the interaction between orientation and elongation type was due to differences between object types at $180^{\circ}$. As Figure 1 illustrates, the participants were quicker to name wide objects at $180^{\circ}$ than either tall objects or non-elongated objects.

Mean naming times for Block 1. A two-way ANOVA of mean naming times for Block 1 showed a large effect of orientation $\left[F(3,141)=12.3, M S_{\mathrm{e}}=26,479, p<.0001\right]$, with a linear component $[F(1,47)=30.5, p<.0001]$ and a quadratic component $[F(1,47)=18.7, p=.0002]$. There was a main effect of object elongation type $[F(2,47)=$ $\left.11.9, M S_{\mathrm{e}}=15,381, p<.0001\right]$. Planned contrasts revealed that wide objects $(M=910.75 \mathrm{msec})$ were named more quickly than tall objects $[M=972.5 \mathrm{msec} ; F(1,47)=$ $20.82, p<.001]$. There was no interaction between orientation and object elongation type for orientations of $0^{\circ}-180^{\circ}[F(6,282)=0.54, p=.78]$ or for orientations of $0^{\circ}-120^{\circ}[F(4,188)=13, p=.96]$. This suggests that the axis of elongation does not influence the process of normalization when rotated objects are first named. In addition, there were no differences between the slopes (orientations, $0^{\circ}-120^{\circ}$ ) for each of the object types (wide objects $=1,122 \mathrm{deg} / \mathrm{sec}$, standard error $[S E]=0.247$; tall objects $=1,125 \mathrm{deg} / \mathrm{sec}, S E=0.253$; and non-elongated objects $=1,104 \mathrm{deg} / \mathrm{sec}, S E=0.237 ; F<1$ ). These slopes are within the previously reported ranges for naming rotated objects of 400-1,400 deg/sec (from Jolicœur 1985; Murray, 1995)

Percentages of error for orientations of $0^{\circ}-\mathbf{1 8 0}^{\circ}$. A three-way repeated measures ANOVA of the error data, with block (4), orientation (4), and object elongation type (3), produced main effects of block $[F(3,141)=3.33$, $\left.M S_{\mathrm{e}}=33.43, p=.02\right]$ and orientation $[F(3,141)=$ $\left.21.655, M S_{\mathrm{e}}=25.68, p<.0001\right]$, but no main effect of object elongation type $\left[F(3,94)=2.547, M S_{\mathrm{e}}=42.12\right.$, $p=.08]$. These results demonstrated that errors decreased over successive blocks and increased as orientations moved away from upright (see Table 1). There was a three-way interaction between block, orientation, and object type $\left[F(18,846)=1.706, M S_{\mathrm{e}}=35.28, p=.03\right]$. Trend analyses revealed that this effect was due to higher order interactions that are uninterpretable. An ANOVA for errors in Block 1 gave a main effect of orientation $\left[F(3,141)=12.3, M S_{\mathrm{e}}=26,479, p<.0001\right]$, where er- 


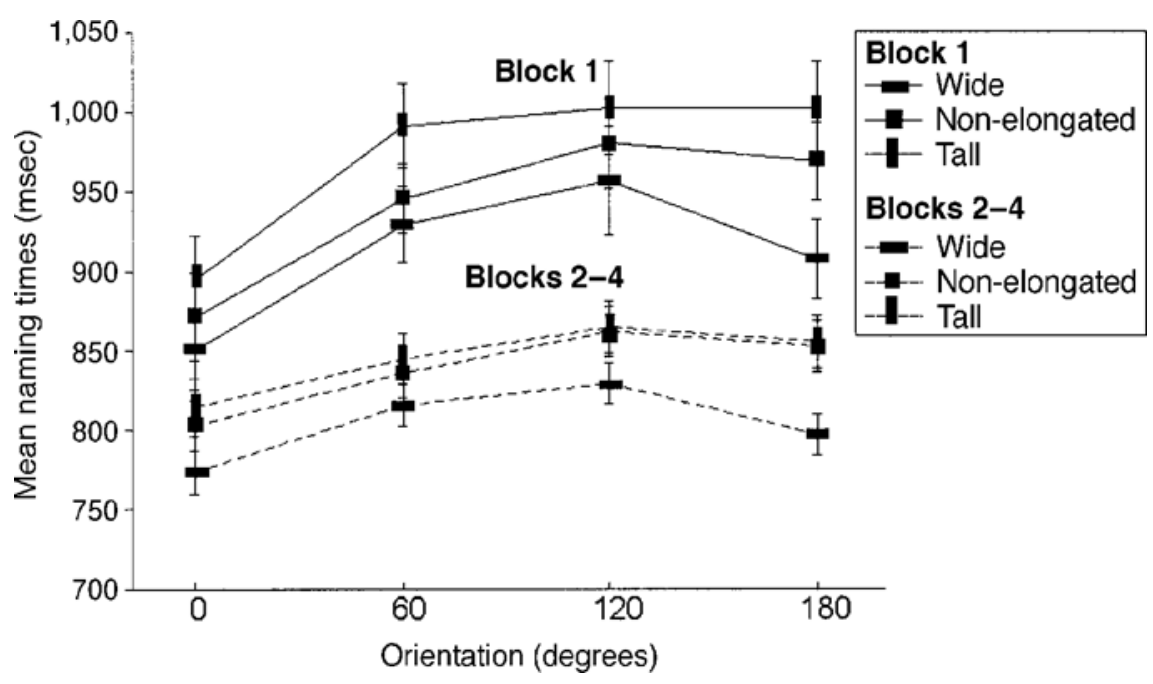

Figure 1. Mean naming times (in milliseconds) and standard errors for wide, non-elongated, and tall objects presented at multiple orientations for Block 1 and collapsed across Blocks 2-4 in Experiment 1.

rors increased as orientations moved away from upright. No other effects were significant. The pattern of errors was not consistent with a speed/accuracy tradeoff.

\section{Discussion}

Theories that implicate the axis of elongation in normalizing rotated images through the shortest angular distance to the upright in order to map them onto long-term object representations appear to be undermined by the lack of significant interactions between orientation and object elongation type in the analyses for orientations between $0^{\circ}$ and $120^{\circ}$ and between $0^{\circ}$ and $180^{\circ}$ on Block 1 (Jolicœur, 1990; Marr, 1982; Marr \& Nishihara, 1978; Tarr \& Pinker, 1990; Ullman, 1989). Perhaps this axis is not the image attribute used to guide orientation normalization in a bottom-up manner. Alternatively, the image may not be rotated prior to accessing a long-term object representation (Biederman, 1987; Corballis, 1988; Hamm $\&$ McMullen, 1998). Similar conclusions have been drawn from a rotated object naming experiment that showed no effect of precuing object orientation on effects of orientation (McMullen et al., 1995; see, also, Gauthier \& Tarr, 1997; Gibson \& Peterson, 1994). McMullen et al. concluded that either finding the top of a rotated object played no part in orientation normalization or objects were normalized before the top of rotated objects was determined.

In contrast, the axis of elongation appeared to influence the attenuation of orientation effects after repeated naming, given the significant interaction between orientation and object elongation type for orientations of $0^{\circ}-180^{\circ}$ in the analysis of all blocks. However, as Figure 1 illustrates, this interaction is due to differences in naming times that occurred at $180^{\circ}$ only. Hence, no significant interactions between orientation and object elongation type were found for orientations between $0^{\circ}$ and $120^{\circ}$.

In addition, wide objects were named more quickly at all orientations and particularly at $180^{\circ}$ than were either tall or non-elongated objects. This effect at $180^{\circ}$ sug-

Table 1

Mean Percentages of Error and Standard Deviations for Wide, Non-Elongated, and Tall Objects Presented at Multiple Orientations for Block 1 and Collapsed Across Blocks 2-4 in Experiment 1

\begin{tabular}{|c|c|c|c|c|c|c|c|c|}
\hline \multirow[b]{2}{*}{ Object Type } & \multicolumn{4}{|c|}{ Orientation for Block 1} & \multicolumn{4}{|c|}{ Orientation for Blocks 2-4 } \\
\hline & $0^{\circ}$ & $60^{\circ}$ & $120^{\circ}$ & $180^{\circ}$ & $0^{\circ}$ & $60^{\circ}$ & $120^{\circ}$ & $180^{\circ}$ \\
\hline \multicolumn{9}{|l|}{ Wide } \\
\hline Mean & 1.11 & 3.85 & 2.57 & 4.10 & 3.06 & 0.93 & 1.07 & 3.86 \\
\hline$S D$ & 4.37 & 8.42 & 7.19 & 7.63 & 3.83 & 2.09 & 2.52 & 4.31 \\
\hline \multicolumn{9}{|l|}{ Non-elongated } \\
\hline Mean & 2.50 & 1.46 & 0.70 & 4.72 & 2.07 & 0.37 & 0.81 & 2.71 \\
\hline$S D$ & 7.03 & 4.91 & 3.37 & 9.52 & 3.84 & 1.46 & 1.98 & 4.01 \\
\hline \multicolumn{9}{|l|}{ Tall } \\
\hline Mean & 1.88 & 0.70 & 3.44 & 3.20 & 1.88 & 1.04 & 1.20 & 2.44 \\
\hline$S D$ & 5.58 & 3.37 & 7.33 & 6.74 & 3.14 & 2.19 & 2.38 & 3.81 \\
\hline
\end{tabular}


gests that it is not the axis of elongation per se, but something particular to objects with long horizontal axes presented at $180^{\circ}$, that quickens naming times both on first naming and after experience in naming specific exemplars. This phenomenon may be due to wide objects at $180^{\circ}$ being rotated out of the picture plane. Murray (1997) demonstrated that rotations out of the picture plane (flipping) resulted in smaller effects of orientation for objects presented at $180^{\circ}$ than did rotation within the plane. Since flipping from $180^{\circ}$ to upright occurs about the horizontal axis, the elongation of this axis may make it more salient, thereby promoting the use of a flipping strategy.

In summary, the results from Experiment 1 did not support the view that axes of elongation, in isolation, influence the normalization of object images during rotated object naming. They did suggest that wide objects were processed more efficiently than tall or non-elongated objects, particularly at the $180^{\circ}$ orientation. However, some caution in interpreting the results is necessary, since symmetry was not controlled for in the choice of stimuli. There is evidence that cues of elongation and symmetry may be processed interactively (Gerhardstein \& Peterson, 1995; Sekuler \& Swimmer, 2000), so it is possible that uncontrolled symmetry cues may have influenced the outcome. This issue is dealt with in Experiment 3 .

\section{EXPERIMENT 2}

On the whole, Experiment 1 did not support a role for axes of elongation on effects of orientation for rotated objects during naming. However, the axis of symmetry has also been implicated in this role (Marr, 1982; Marr \& Nishihara, 1978; Tarr \& Pinker, 1990). To test this hypothesis, rotated, symmetrical, and asymmetrical objects were named.

Tarr and Pinker (1990) found smaller effects of orientation on the identification of rotated, symmetrical nonsense objects than on the identification of asymmetrical versions of similar objects. McMullen and Farah (1991) tested the generality of these findings with respect to the naming of rotated real objects. They reported that effects of orientation diminished more for repeated naming of rotated symmetrical than of asymmetrical real objects. However, symmetry did not influence effects of orientation during the first block of naming. McMullen and Farah's study was not designed to test effects of symmetry on orientation and so relied on a post hoc analysis of responses to unequal numbers of symmetrical and asymmetrical objects presented at each orientation. The noise inherent in these conditions, combined with the uncertainty associated with first-time naming relative to practiced naming, could have masked effects of symmetry on orientation during the first block of naming. McMullen and Farah also relied on their own subjective ratings of symmetry.

In the experiment reported here, independentobservers rated objects on a 5-point scale with very symmetrical and very asymmetrical as end points. Equal numbers of asymmetrical and symmetrical objects, as determined by this rating, were named at each of six orientations to determine the influence of a symmetrical axis on rotated object naming.

\section{Method}

Participants. Forty-eight undergraduates at Dalhousie University participated for monetary or class credit. All the participants had normal or corrected-to-normal vision and reported English as their first language. None of the participants had participated in Experiment 1.

Apparatus and Stimuli. Stimuli were presented using the equipment described in Experiment 1. Twenty participants who did not participate in any part of Experiment 1 or in the naming trials of this experiment rated 171 objects from the Snodgrass and Vanderwart (1980) set for symmetry on a 5-point scale. Ratings were based on the particular view of the object depicted, rather than on whether the object could be considered to be symmetrical in any view. From these rated objects, 72 were selected for presentation during the naming trials. Half were rated as symmetrical, and half were rated as asymmetrical (see Appendix B for mean symmetry ratings per object). (N.B.: Only the "Moon," an object not used in the naming experiment, was symmetrical about the $x$-axis. All of the objects that were named were symmetrical about the $y$-axis.)

Procedure. The procedure was similar to that used in Experiment 1 , except that 6 objects were shown at each of six orientations: $0^{\circ}, 60^{\circ}, 120^{\circ}, 180^{\circ}, 240^{\circ}$, and $300^{\circ}$. Six random orders of 72 trials each were created with the same constraints as those imposed on the orders created for Experiment 1. Each participant named six blocks of 72 objects, half of which were symmetrical and half of which were not. Block orders were shown in a Latin square across all participants such that all the participants saw each object in each orientation an equal number of times. Since there was no statistical difference between mean naming times for orientations of $60^{\circ}$ and $300^{\circ}$ and for orientations of $120^{\circ}$ and $240^{\circ}$, these orientations were collapsed for further analysis, resulting in twice as many presentations for orientations of $60^{\circ}$ and $120^{\circ}$ as for orientations of $0^{\circ}$ and $180^{\circ}$.

\section{Results}

Mean naming times for all blocks. Two repeated measures ANOVAs were performed. These analyses were conducted on data for orientations of $0^{\circ}-180^{\circ}$, with block (6), orientation (4), and symmetry (2) as within-subjects factors, and for orientations of $0^{\circ}-120^{\circ}$, with factors of block (6), orientation (3), and symmetry (2). As in Experiment 1 , only the results of the analysis for orientations of $0^{\circ}-180^{\circ}$ will be reported, unless the results of the analysis for $0^{\circ}-120^{\circ}$ differ in significance. There was a main effect of block $\left[F(5,235)=14.1, M S_{\mathrm{e}}=45,272.9\right.$, $p<.0001]$, indicating a decrease in naming times as a result of practice. Large effects of orientation were found on the time to name rotated objects $[F(3,141)=47.7$, $\left.M S_{\mathrm{e}}=5,763, p<.0001\right]$. A trend analysis revealed a linear component $[F(1,47)=88.52, p<.0001]$ and a quadratic component $[F(1,47)=14.91, p=.0006]$ to this effect for orientations of $0^{\circ}-180^{\circ}$ and a linear component only for orientations of $0^{\circ}-120^{\circ}[F(1,47)=16.5, p=$ $.0004]$. There was a main effect of symmetry $[F(1,47)=$ $\left.29.9, M S_{\mathrm{e}}=14,716.9, p<.0001\right]$. As is illustrated in Figure 2, overall, symmetrical objects $(M=772 \mathrm{msec})$ were named more quickly than asymmetrical objects $(M=$ 
$799 \mathrm{msec})$. There was an orientation $\times$ symmetry interaction $[F(3,141)=5.99, p=.0007]$ for orientations between $0^{\circ}$ and $180^{\circ}$, with a linear component $[F(1,47)=$ $12.9, p=.0011]$ that was not present for orientations of $0^{\circ}$ to $120^{\circ}$, indicating that there was an effect of symmetry on orientation only at $180^{\circ}$. The interaction between orientation and block was not significant for either orientations of $0^{\circ}-180^{\circ}$ or orientations of $0^{\circ}-120^{\circ}$. However, there was an interaction between block, orientation, and symmetry for orientations of $0^{\circ}-180^{\circ}[F(15,705)=1.7$, $p=.03]$ and orientations of $0^{\circ}-120^{\circ}[F(10,470)=2.5, p=$ .007], suggesting that effects of orientation diminished at different rates during the four blocks in relation to symmetry and asymmetry.

To investigate this three-way interaction further, separate ANOVAs were performed on symmetrical objects and asymmetrical objects, each with repeated measures on factors of block (6) and orientation (4). The analysis of symmetrical objects showed a block $\times$ orientation interaction for orientations of $0^{\circ}-180^{\circ}[F(15,705)=1.679$, $p=.05]$ and for orientations of $0^{\circ}-120^{\circ}[F(10,470)=$ $2.1, p=.02]$. This interaction was not present for asymmetrical objects in the analysis of orientations between $0^{\circ}$ and $180^{\circ}$. In the analysis of asymmetrical objects for orientations of $0^{\circ}-120^{\circ}$, a block $\times$ orientation interaction did occur $[F(10,470)=1.9, p=.05]$. However, as is shown in Figure 2, orientation effects diminished more strongly for symmetrical objects than for asymmetrical objects at $180^{\circ}$.

In a related analysis, the slopes at orientations of $0^{\circ}-120^{\circ}$ for asymmetrical and symmetrical objects in Block 1 were compared with the average slopes for Blocks 2-6. Symmetrical objects showed the greatest slope difference after repeated naming (Block $1=$ 1,832 deg/sec, Blocks 2-6 = 3,226 deg/sec, slope difference $=1,394 \mathrm{deg} / \mathrm{sec}$, vs. Block $1=1,709 \mathrm{deg} / \mathrm{sec}$, Blocks $2-6=2,545 \mathrm{deg} / \mathrm{sec}$, slope difference $=836 \mathrm{deg}$ / $\mathrm{sec}$ for asymmetrical objects). A $t$ test comparing Block 1 slopes and the average for the slopes of Blocks 2-6 approached significance for symmetrical objects $[t(1,47)=$ $1.69, p=.09]$. This suggested that the effects of orientation diminished across blocks more for symmetrical objects than for asymmetrical objects.

Mean naming times for Block 1. A two-way ANOVA of mean naming times for Block 1 showed a large effect of orientation $\left[F(3,141)=7.8, M S_{\mathrm{e}}=16,894, p<.0001\right]$, with a linear component $[F(1,47)=30.5, p<.0001]$, a quadratic component $[F(1,47)=18.7, p=.0002]$, and a linear component only for orientations between $0^{\circ}$ and $120^{\circ}[F(1,47)=36.6, p<.0001]$. There was a main effect of symmetry $\left[F(1,47)=7.9, M S_{\mathrm{e}}=21,855, p=\right.$ .0072]. As is illustrated in Figure 2, symmetrical objects were named faster than asymmetrical objects. There was no interaction between orientation and symmetry for orientations of $0^{\circ}-180^{\circ}(F<1)$ or for orientations of $0^{\circ}-120^{\circ}$ $(F<1)$. In addition, there were no differences between the slopes for asymmetrical $(1,709 \mathrm{deg} / \mathrm{sec}, S E=0.222)$ and symmetrical $(1,832 \mathrm{deg} / \mathrm{sec}, S E=0.173)$ objects at orientations of $0^{\circ}-120^{\circ}(t<1)$. This suggests that the axis of symmetry did not influence the process of normalization when rotated objects were first named.

Percentages of error for orientations of $0^{\circ}-\mathbf{1 8 0}^{\circ}$. Analysis of the accuracy data (see Table 2) produced main effects of block $\left[F(5,235)=25.4, M S_{\mathrm{e}}=45.5, p<\right.$ $.0001]$ and orientation $\left[F(3,141)=2.8, M S_{\mathrm{e}}=29.4, p=\right.$ $.04]$, where errors decreased over block and increased as orientations moved away from upright. There were no significant effects from the analysis of errors in Block 1. The pattern of errors for the overall analysis and for Block 1 was inconsistent with a speed/accuracy tradeoff.

\section{Discussion}

In an experiment designed to test the effects of orientation on naming symmetrical and asymmetrical line

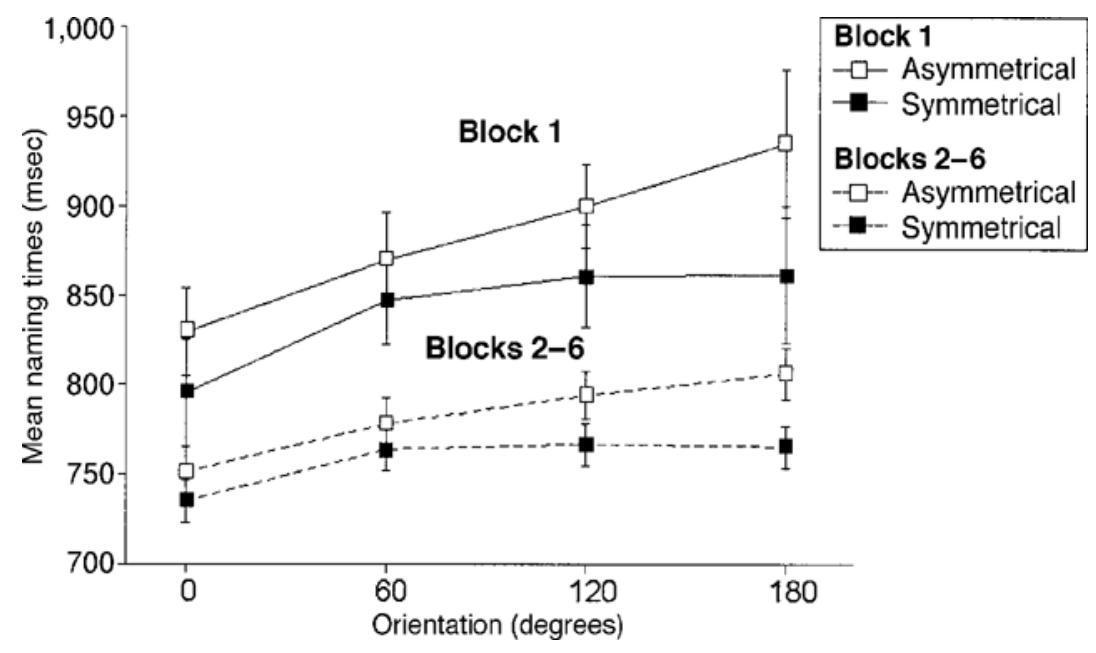

Figure 2. Mean naming times (in milliseconds) and standard errors for asymmetrical and symmetrical objects presented at multiple orientations for Block 1 and collapsed across Blocks 2-6 in Experiment 2. 
Table 2

Mean Percentages of Error and Standard Deviations for Asymmetrical and Symmetrical Objects Presented at Multiple Orientations for Block 1 and Collapsed Across Blocks 2-6 in Experiment 2

\begin{tabular}{|c|c|c|c|c|c|c|c|c|}
\hline \multirow[b]{2}{*}{ Object Type } & \multicolumn{4}{|c|}{ Orientation for Block 1} & \multicolumn{4}{|c|}{ Orientation for Blocks 2-6 } \\
\hline & $0^{\circ}$ & $60^{\circ}$ & $120^{\circ}$ & $180^{\circ}$ & $0^{\circ}$ & $60^{\circ}$ & $120^{\circ}$ & $180^{\circ}$ \\
\hline \multicolumn{9}{|l|}{ Asymmetrical } \\
\hline Mean & 4.52 & 6.63 & 6.98 & 5.28 & 1.39 & 1.48 & 1.91 & 1.67 \\
\hline$S D$ & 8.24 & 10.71 & 11.55 & 8.64 & 2.16 & 2.07 & 2.58 & 2.38 \\
\hline \multicolumn{9}{|l|}{ Symmetrical } \\
\hline Mean & 5.42 & 4.57 & 6.36 & 8.13 & 1.56 & 1.92 & 1.88 & 2.08 \\
\hline$S D$ & 10.67 & 8.52 & 11.00 & 13.85 & 2.42 & 2.87 & 3.11 & 3.40 \\
\hline
\end{tabular}

drawings of real objects, no interaction was found between orientation and symmetry in the analysis for Block 1, demonstrating that there was no difference in orientation sensitivity to symmetrical and asymmetrical objects on first naming. This result undermines theories of object identification postulating that the axis of symmetry is used to guide normalization of rotated objects to the upright (Jolicœur, 1990; Marr, 1982; Marr \& Nishihara, 1978; McMullen \& Jolicœur, 1992; Tarr \& Pinker, 1990). As was discussed in Experiment 1, this result could occur simply because the axis of symmetry is not an appropriate object attribute used to guide normalization in a bottom-up manner. Alternatively, normalization may not rely on perceptual attributes of the stimulus, such as axes, because top-down information obtained from representations contacted prior to normalization could subsequently guide normalization (Biederman, 1987; Corballis, 1988; Hamm \& McMullen, 1998).

Consistent with McMullen and Farah's (1991) findings, symmetrical objects demonstrated a greater diminishment in effects of orientation, as compared with asymmetrical objects. This result suggests that effects of symmetry influenced effects of orientation only after practice with the object set.

According to Tarr and Pinker (1990), object-centered representations can encode parts and their spatial relations only along one dimension. Since the parts for twodimensional symmetrical objects are repeated about the axis of symmetry, the visual system can access an orientation-invariant object-centered representation. The greater reduction in orientation effects for symmetrical objects may be explained by access to orientation-invariant object-centered representations after repeated exposure.

An alternative explanation could be that after repeated naming, objects are more efficiently recognized on the basis of orientation-invariantfeatures/attributes. A featurebased system processes local features of an object, extracting shape and surface attributes, of which some are orientation invariant. With experience, participants learn to associate these orientation-invariant features with some of the objects, resulting in reduced effects of orientation (Jolicœur, 1990; Jolicœur \& Humphrey, 1998). Murray, Jolicœur, McMullen, and Ingleton (1993) demonstrated that with line drawings of common objects, the reduction of orientation effects resulting from repeated naming of objects at one set of orientations transferred to a new "surprise" set of orientations. This supports the notion that the analysis of local orientation-invariant features of objects underlies the attenuation of orientation effects for common objects. Local features of symmetrical objects are mirrored about their axis, resulting in feature redundancy. It may be that this built-in redundancy of features leads to greater attenuation of orientation effects. This explanation is supported by the fact that symmetrical objects, overall, were named more quickly and efficiently than asymmetrical objects.

In summary, the results from Experiment 2 failed to show an influence of symmetry on orientation during the first block of naming. In contrast, with repeated exposure to the object set, effects of orientation diminished more for symmetrical than for asymmetrical objects, as was found by McMullen and Farah (1991). This result only approached significance, however. Experiment 2 did not control for effects of elongation. In Experiment 3 , we controlled for axes of elongation and found a significant difference in the reduction of orientation effects for symmetrical objects, as compared with asymmetrical objects.

\section{EXPERIMENT 3}

The results from Experiments 1 and 2 suggested that axes of elongation or symmetry did not influence normalization the first time that rotated objects were named. However, these axes were examined in isolation, and Sekuler and Swimmer (2000) demonstrated that these axes are processed interactively by the visual system. In an unpublished preliminary investigation, Gerhardstein and Peterson (1995) examined the influence of the axes of elongation and symmetry on orientation when rotated line drawings were named. They found that neither of these axes influenced orientation effects, but they did find that for stimuli with vertical axes of elongation, symmetrical objects were named more quickly than asymmetrical objects. However, for objects with horizontal axes of elongation, asymmetrical objects were named more quickly than symmetrical objects. They concluded that the axis of elongation influenced object identification only in combination with an axis of symmetry and that neither axis had an influence on effects of 
orientation. In the present experiment, the axes of elongation and symmetry were controlled orthogonally, as they were by Gerhardstein and Peterson. One half of the objects were symmetrical, of which half had elongated horizontal axes (wide) and half had elongated vertical axes (tall). The other half of the objects were asymmetrical, of which half were classified as tall and half as wide objects. This design allowed for the examination of a possible interdependence between the two types of axes. ${ }^{1}$ For each group of objects, name frequency was controlled.

A second methodological change from the previous experiments was the method used to define the axes of elongation and symmetry. Previously, these axes were defined on the basis of observer ratings. In the following Experiment 3, objects were defined as elongated if their aspect ratios were above 7:5 and as symmetrical if the left-hand side mirrored the right-hand side exactly. To calculate aspect ratio, each object was measured along its vertical and horizontal axes. In addition, since Sekuler (1996; Sekuler \& Swimmer, 2000) reported that the effects of elongation increased with increases in aspect ratio, all the objects were divided into two groups, depending on whether their aspect ratio fell above or below a cutoff of $2: 1$. By $2: 1$, we mean that, for tall objects, the vertical axis was twice that of the horizontal axis and, for wide objects, the horizontal axis was twice that of the vertical axis. Finally, in Experiment 3, objects at $180^{\circ}$ of rotation were not included in the design, because the process of normalization within the plane may not be utilized at this orientation (Jolicœur, 1990; Jolicour \& Humphrey, 1998; Murray et al., 1993). The omission of the $180^{\circ}$ orientation also allowed for an increase in the number of objects for the remaining orientations. It was expected that if the axes were used interactively in the naming of rotated objects, there would be a three-way interaction between the factors of orientation, elongation, and symmetry when first naming objects and after repeated naming. Finally, since increasing the aspect ratio of an object increased the salience of the axis of elongation, we predicted that increasing the aspect ratio would influence the effects of orientation.

\section{Method}

Participants. Fifty-six undergraduates with normal or correctedto-normal vision (40 females, 16 males), between 18 and 29 years of age, volunteered or received course credit for their participation. Two participants failed to meet a performance criterion of $60 \%$ correct in every cell of the design and an overall performance of $70 \%$ correct. Their data were excluded from statistical analysis. No student participated in any other experiment reported here.

Stimuli and Apparatus. Ninety-six line drawings of common objects were selected for presentation. Sixty-nine were taken from Snodgrass and Vanderwart (1980), of which 33 were altered to conform to experimental requirements, and 27 were taken from photographs and children's drawing books. Half of the drawings were asymmetrical, and half were symmetrical, about the $y$-axis. The left and right sides of the symmetrical drawings were exact mirror images of each other. The majority of asymmetrical drawings were chosen from the set of objects rated in Experiment 2 as asymmetrical
$(M=4.5, S D=0.39)$. Within each of these two sets, half contained a horizontal axis of elongation (wide objects), and half contained a vertical axis of elongation (tall objects). Examples of the stimuli from each condition are shown in Figure 3. The aspect ratios of elongated objects ranged between 7:5 and 3:1. The four groups of drawings (tall asymmetrical, wide asymmetrical, tall symmetrical, and wide symmetrical) were divided in half, with 12 objects having aspect ratios below 2:1 and 12 objects having aspect ratios above $2: 1$. Each of these four groups of drawings were matched for name frequency (Kučera \& Francis, 1967): tall asymmetrical $(M=21.5)$, wide asymmetrical $(M=25.9)$, tall symmetrical $(M=24.5)$, and wide symmetrical $(M=31)$. There was no difference between the name frequency of the three groups $[F(3,23)=0.37, p=.78]$. Line drawings were presented as black lines on a white background, with an average visual angle of $5^{\circ}$, in the center of a 17 -in. MacIntosh G3 monitor. The experiment was controlled by a custom program (Christie, 1999).

Procedure. Each experimental session consisted of six blocks of 96 trials. Within each block, 24 trials were tall symmetrical objects, 24 were wide symmetrical objects, 24 were tall asymmetrical objects, and 24 were wide asymmetrical objects. These four sets of 24 stimuli were further divided by orientation; in each set of 24 stimuli, 8 objects were shown at $0^{\circ}$, and the remaining 16 objects were divided evenly and shown at orientations of $60^{\circ}, 120^{\circ}, 240^{\circ}$, and $300^{\circ}$, respectively. Naming times for objects shown at $60^{\circ}$ and $300^{\circ}$ and objects shown at $120^{\circ}$ and $240^{\circ}$ were collapsed for analysis. No object was repeated during a single block. This procedure resulted in each participant's seeing 8 objects at each of three orientations (with $60^{\circ}$ and $300^{\circ}$ collapsed and $120^{\circ}$ and $240^{\circ}$ collapsed) in every block. The same 96 stimulus objects were seen at different orientations across the six blocks, so that each object was shown twice at $0^{\circ}$ and once at all other orientations. The trials within each block were presented in random order. The six blocks were presented in a pseudorandom sequence such that, during the course of the experiment, each block occurred nine times in each of the six possible positions within the sequence of six blocks. For example, Block 1 was the first block for 9 participants, the second block for another 9 participants, and so on. This ensured that every object at every orientation was seen an equal number of times at every position within the sequence of six blocks. The trials were initiated when the experimenter pressed a computer key. A trial consisted of two frames. First, a fixation cross appeared in the center of the screen for $500 \mathrm{msec}$, and then, following an interstimulus interval of $500 \mathrm{msec}$, a stimulus object appeared. The observer's task was to name the object presented on the screen as quickly as possible. Vocal responses were timed by the program as threshold changes to the sound channel, which resulted in the stimulus object's disappearing from the screen. If no response was made, the stimulus object automatically disappeared after $2 \mathrm{sec}$. The experimenter keyed in whether the observer's response was correct, incorrect, or spoiled. Responses were judged correct according to the criteria laid out in Appendix C. A trial was considered spoiled if the threshold of the sound channel was not reached or was reached prematurely (i.e., an observer coughed or said "er") or if the observer failed to respond in $2 \mathrm{sec}$. Spoiled trials were not included in the analyses. The observers were given 14 practice trials with objects presented randomly at each of five orientations. Objects named in the practice trials did not appear in the experimental trials.

\section{Results}

Mean naming times and accuracy for the factors of block (6), orientation (3), elongation (2), symmetry (2), and salience (2) were analyzed in three separate repeated measures ANOVAs. The first analysis included block, orientation, elongation, and symmetry as repeated factors. The second analysis examined the results for the 


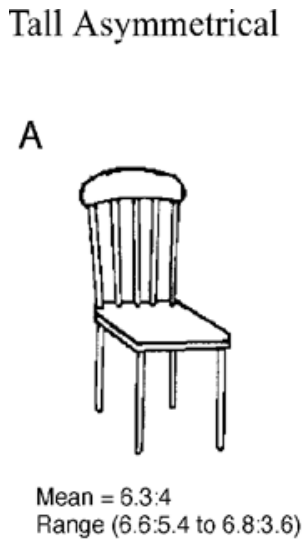

B

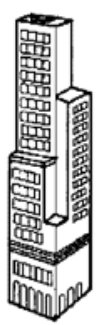

Mean $=6 \cdot 9 \cdot 2 \cdot 6$

Range (7.3:3.6 to $7.3: 1.4)$

\section{Tall Symmetrical}

Congruent

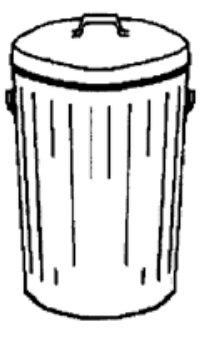

Mean $=6 \cdot 6: 3.9$

Range (6.6.5.4 to $7.0: 3.6)$

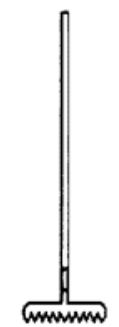

Mean $=7 \cdot 1: 2.6$

Range (7.1:3.5 to $7.2: 1.8$ )
Mean $=3.8: 7$

Range ( $4: 6.8$ to $3.4: 6.6)$
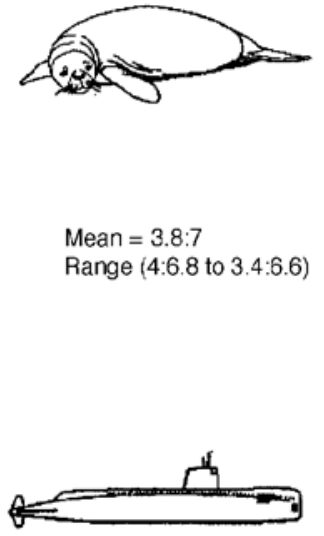

Mean $=7 \cdot 1: 2 \cdot 6$

Range (3:6.6 to $1.8: 7)$
Wide Symmetrical

\section{Incongruent}

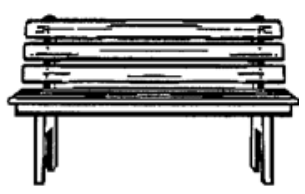

Mean $=3.9: 7$

Range (4:6.9 to $3.7: 7.1)$

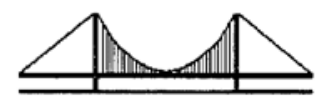

Mean $=7.1: 2.2$

Range (3:7.1 to 1.5:7.1)

Figure 3. Examples of stimuli used in Experiment 3: (A) objects whose aspect ratios are below 2:1 and (B) objects whose aspect ratios are above $2: 1$. The mean and range of the aspect ratios are listed for each object set. Tall and wide asymmetrical objects defined only an axis of elongation. Tall symmetrical objects defined congruent axes of symmetry and elongation. Wide symmetrical objects defined incongruent axes of symmetry and elongation.

first block only, with orientation (3), elongation (2), and symmetry (2) as repeated factors. The third analysis included orientation (3), elongation (2), and salience (2) as factors. The salience factor was not included in a fiveway ANOVA, since it did not balance across block and orientation or pertain to symmetry.

Mean naming times for all blocks. Mean naming times and percentages correct were analyzed with a fourway repeated measures ANOVA with block (6), orientation (3), elongation (2), and symmetry (2) as within-subjects factors. There was a main effect of block $[F(5,285)=$ $\left.121.7, M S_{\mathrm{e}}=24,591, p<.0001\right]$, signifying that naming times decreased with practice. Large effects of object orientation were found $\left[F(2,106)=136.5, M S_{\mathrm{e}}=7,892\right.$, $p<.0001]$. A trend analysis revealed a linear component to this effect $[F(1,53)=223.9, p<.0001]$. There was a main effect of symmetry $\left[F(1,53)=23.7, M S_{\mathrm{e}}=28,570\right.$, $p<.0001]$, with symmetrical objects $(M=895 \mathrm{msec})$ being named faster than asymmetrical objects $(M=$ $922 \mathrm{msec})$, and a main effect of elongation $[F(1,53)=19.1$, $\left.M S_{\mathrm{e}}=11,395.9, p<.0001\right]$, with tall objects $(M=$ $901 \mathrm{msec})$ being named faster than wide objects $(M=$ $916 \mathrm{msec}$ ). There was an interaction between block and orientation $[F(10,530)=4.3, p<.0001]$, indicating that effects of orientation diminished with repeated naming.
There was also an interaction between symmetry and elongation $[F(1,53)=4.5, p=.04]$, whereby symmetry affected wide objects more than it did tall objects.

More important for the theory tested here, there were no two-way interactions between orientation and elongation or orientation and symmetry. However, there was a three-way interaction between these variables $[F(2,106)=$ $3.4, p=.036]$, which had a linear component $[F(1,53)=$ $5.6, p=.02]$. As Figure 4 illustrates, this interaction centered on tall objects, with tall asymmetrical objects demonstrating more sensitivity to orientation $(1,650 \mathrm{deg} / \mathrm{sec}$, $S E=0.082)$ than did tall symmetrical objects [3,268 deg/ $\mathrm{sec}, S E=0.065 ; t(1,323)=3.04, p=.0026]$. There was no reliable difference between the slopes for wide asymmetrical objects $(2,119 \mathrm{deg} / \mathrm{sec}, S E=0.067)$ and for wide symmetrical objects (2,000 deg/sec, $S E=0.074 ; t<1$; a power analysis gave a value of 0.059$)$. Note that the tall symmetrical objects were congruent with respect to the two axes that they defined and were least affected by orientation $(3,268 \mathrm{deg} / \mathrm{sec})$. In contrast, the wide symmetrical objects were incongruent with respect to the axes that they defined and were more affected by orientation $(2,000 \mathrm{deg} / \mathrm{sec})$. A $t$ test comparing the slopes of tall symmetrical objects and wide symmetrical objects was significant $[t(1,323)=1.898, p=.05]$. These results in- 


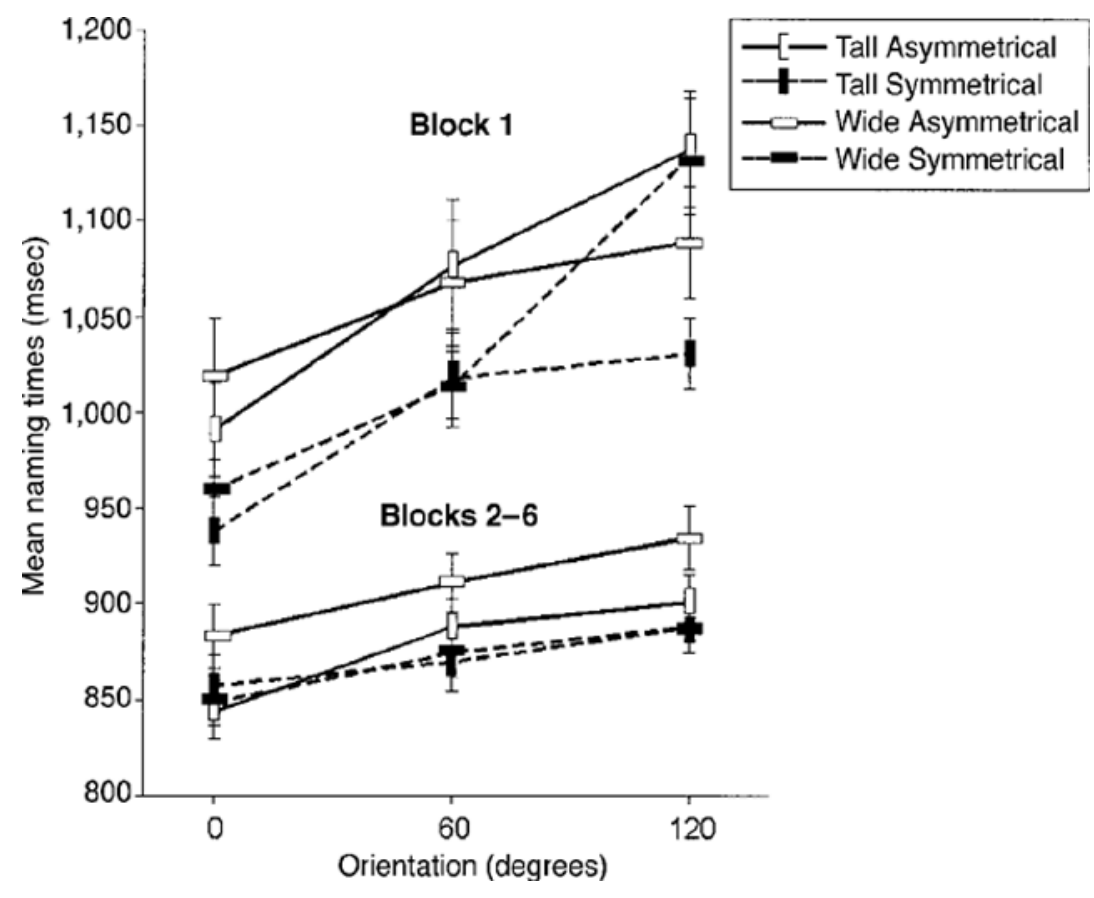

Figure 4. Mean naming times (in milliseconds) and standard errors for tall asymmetrical, tall symmetrical, wide asymmetrical, and wide symmetrical objects presented at multiple orientations for Block 1 and collapsed across Blocks 2-6 in Experiment 3.

dicated that for objects with vertical axes of elongation, the property of vertical symmetry facilitated the processing of rotated images but that this facilitation did not extend to objects with horizontal axes of elongation. Finally, there was a four-way interaction between block, orientation, elongation, and symmetry $[F(10,530)=$ $1.981, p=.03$ ], suggesting that the diminishment of orientation effects in the four groups of objects occurred at different rates. The slopes for each of the conditions across Blocks 1-6 demonstrated that the diminishment of orientation effects was least for wide asymmetrical objects and greatest for wide symmetrical objects. A comparison of slopes for Block 1 versus the average slopes for Blocks 2-6 showed that slope differences were greatest for conditions with symmetrical objects [tall symmetrical slope difference $=2,619 \mathrm{deg} / \mathrm{sec}, t(1,53)=$ $2.493, p=.0158$; wide symmetrical slope difference $=$ $2,412 \mathrm{deg} / \mathrm{sec}, t(1,53)=4.021, p=.0002 ;$ tall asymmetrical slope difference $=1,326 \mathrm{deg} / \mathrm{sec}, t(1,53)=$ $2.457, p=.0173$; and wide asymmetrical slope differences $=650 \mathrm{deg} / \mathrm{sec}, t<1]$. These slope differences suggest that symmetry facilitated the diminishment of orientation effects after repeated naming for orientations between $0^{\circ}$ and $120^{\circ}$, similar to the finding in Experiment 2.

Percentages of error for all blocks. A four-way repeated measures analysis of error, including block (6), orientation (3), elongation (2), and symmetry (2) as factors, was performed. Main effects of block $[F(5,265)=$ $73.1, p<.0001]$ and orientation $[F(2,106)=4.2, p=$ $.02]$ indicated that errors decreased over blocks and in- creased as objects were rotated further from upright (see Table 3). There was also a main effect of symmetry $[F(1,53)=13.6, p=.0005]$, with fewer errors for symmetrical objects than for asymmetrical objects. There was an interaction between elongation and symmetry $[F(1,53)=31.2, p<.0001]$, with more errors for wide asymmetrical objects than for wide symmetrical objects, but little difference in error rates for tall symmetrical and asymmetrical objects. These results were inconsistent with speed/accuracy tradeoffs.

Mean naming times for Block 1. Mean naming times and percentages correct were analyzed with a three-way repeated measures ANOVA with orientation(3), elongation (2), and symmetry (2) as within-subjects factors. Large effects of orientation were found $[F(2,106)=$ $30.8, p<.0001]$. A trend analysis revealed a linear component to this effect $[F(1,53)=64.4, p<.0001]$. There was a main effect of symmetry $[F(1,53)=24, p<.0001]$, but no effect of elongation. Planned contrasts revealed that symmetrical objects $(M=1,016 \mathrm{msec})$ were named faster than asymmetrical objects $(M=1,063 \mathrm{msec})$. There were no two-way interactions between orientation and elongation or orientation and symmetry; however, there was a two-way interaction between elongation and symmetry $[F(1,53)=5.7, p=.02]$, where the difference in naming times between asymmetrical and symmetrical objects was greater for tall objects than for wide objects. There was also a three-way interaction between orientation, elongation, and symmetry $[F(2,106)=4.3, p=$ $.016]$, which had a linear component $[F(1,53)=6.39$, $p=.014]$. Figure 4 illustrates that symmetrical objects 
Table 3

Mean Percentages of Error and Standard Deviations for Tall Asymmetrical, Tall Symmetrical, Wide Asymmetrical, and Wide Symmetrical Objects Presented at Multiple Orientations for Block 1 and Collapsed Across Blocks 2-6 in Experiment 3

\begin{tabular}{|c|c|c|c|c|c|c|}
\hline \multirow[b]{2}{*}{ Object Type } & \multicolumn{3}{|c|}{ Orientation for Block 1} & \multicolumn{3}{|c|}{ Orientation for Blocks 2-6 } \\
\hline & $0^{\circ}$ & $60^{\circ}$ & $120^{\circ}$ & $0^{\circ}$ & $60^{\circ}$ & $120^{\circ}$ \\
\hline \multicolumn{7}{|c|}{ Tall asymmetrical } \\
\hline Mean & 14.12 & 12.50 & 16.20 & 6.20 & 6.53 & 7.36 \\
\hline$S D$ & 12.86 & 13.52 & 15.29 & 4.36 & 4.92 & 4.99 \\
\hline \multicolumn{7}{|l|}{ Tall symmetrical } \\
\hline Mean & 9.26 & 12.96 & 13.43 & 6.62 & 6.71 & 6.90 \\
\hline$S D$ & 10.36 & 10.57 & 11.86 & 4.27 & 4.53 & 4.55 \\
\hline \multicolumn{7}{|c|}{ Wide asymmetrical } \\
\hline Mean & 10.65 & 9.72 & 14.35 & 5.37 & 4.58 & 5.23 \\
\hline$S D$ & 10.13 & 10.21 & 12.71 & 4.05 & 4.23 & 3.95 \\
\hline \multicolumn{7}{|c|}{ Wide symmetrical } \\
\hline Mean & 16.20 & 13.66 & 17.36 & 8.84 & 8.10 & 8.61 \\
\hline$S D$ & 11.55 & 8.51 & 12.24 & 4.65 & 5.25 & 5.82 \\
\hline
\end{tabular}

were named faster than asymmetrical objects, except for wide objects at $120^{\circ}$, at which point wide asymmetrical objects were named faster than wide symmetrical objects. Analysis of the slopes for each of the four conditions (tall asymmetrical, wide asymmetrical, tall symmetrical, and wide symmetrical) showed that orientation effects were stronger for wide symmetrical objects $(713 \mathrm{deg} / \mathrm{sec})$ than for wide asymmetrical objects $[1,742 \mathrm{deg} / \mathrm{sec}, S E=$ $0.29 ; t(1,53)=1.95, p=.05]$. Tall asymmetrical objects showed stronger orientation effects $(788 \mathrm{deg} / \mathrm{sec})$ than did tall symmetrical objects $(1,349 \mathrm{deg} / \mathrm{sec}, S E=0.19$; $t(1,53)=2.033, p=.05]$. It appeared that vertical symmetry facilitated the identification of objects with congruent vertical axes of elongation but interfered with the identification of objects with incongruent horizontal axes of elongation.

Percentages of error for Block 1. A repeated measures ANOVA for errors, with orientation (3), elongation (2), and symmetry (2) as factors, was performed. There was a main effect of orientation $[F(2,106)=3.242, p=$ $.04]$, where errors increased at orientations further from upright. There was an interaction between elongation and symmetry $[F(1,53)=17.5, p=.0001]$, with more errors for tall asymmetrical objects than for tall symmetrical objects and more errors for wide symmetrical objects than for wide asymmetrical objects. No other effects were significant, indicating no support for speed/accuracy tradeoffs.

Mean naming times for saliency. Mean naming times and percentages correct were analyzed with a three-way repeated measures ANOVA with orientation (3), elongation (2), and salience (2) as within-subjects factors. There was a main effect of salience $[F(1,53)=20.6, p=.0001]$, where objects with short aspect ratios (below 2:1, $M=$ 899.5 ) were named faster than objects with long aspect ratios (above $2: 1, M=918$ ). There was an interaction between elongation and salience $[F(1,53)=12.86, p=$ .0003], where increasing aspect ratio made little difference to the naming of wide objects (short aspect ratio $=$ $913 \mathrm{msec}$ and long aspect ratio $=922 \mathrm{msec}$ ) but tall ob- jects with short aspect ratios were named faster ( $886 \mathrm{msec})$ than tall objects with long aspect ratios (914 msec). These results suggest that increasing the salience of the axis of elongation actually impaired rotated object naming.

There was no significant interaction between orientation and salience, nor were there any three-way interactions between orientation, salience, and elongation, suggesting that this manipulation made no difference to the effects of orientation on naming the four groups of objects.

Percentages of error for saliency. There was no main effect of salience $[F(1,53)=1.56, p=.2177]$. There was a two-way interaction between elongation and salience $[F(1,53)=11.3, p<.0001]$, where more errors were made for wide objects with short aspect ratios than for wide objects with long aspect ratios but the opposite was true for tall objects. There were no interactions involving orientation. These results were consistent with no speed/accuracy tradeoffs.

\section{Discussion}

Similar to the findings from Experiments 1 and 2, no interactions were found between orientation and elongation or orientation and symmetry on the first naming of the objects or after repeated naming. This provides additional support for the view that these axes did not, in isolation, influence the normalization of rotated images.

Rotation from upright always increased naming times. Practice over blocks decreased naming times, and effects of orientation diminished with practice. Symmetry always facilitated naming times. Elongation effects were present only after repeated naming and manifested as an advantage for naming tall over wide objects. In Block 1, symmetry affected tall objects more than it did wide ones. This effect reversed after repeated naming, where symmetry affected wide objects more than it did tall ones. Of greater interest to the theories tested by this experiment were the three-way interactions.

Congruent and incongruent axes effects. When rotated objects were first named, incongruency between 
the axes defined by elongation and symmetry increased effects of orientation. This effect of incongruency was particularly apparent at the most extreme orientation tested, $120^{\circ}$. Figure 4 shows that when the axes of elongation and symmetry were incongruent with each other (wide objects symmetrical about the short axis), effects of orientation were greater, as compared with objects with axes of elongation only (tall and wide asymmetric objects) and for stimuli with congruent axes (tall symmetrical). This pattern of inhibition for objects with incongruent axes of elongation and symmetry will be discussed further in the General Discussion section.

In contrast, after repeated naming, congruency effects were found in an analysis of all blocks. Tall symmetrical objects (where the axes of elongation and symmetry define the same axis) were least affected by orientation, followed by wide symmetrical objects. Clearly, symmetry aided in the diminution of effects of orientation.

As was reported by McMullen and Farah (1991) and in Experiment 2 of this paper, there was greater diminishment of orientation effects for symmetrical objects than for to asymmetrical objects after repeated naming. It appears that the axis of symmetry influenced both orientation effects and overall naming times. The axis of symmetry may not guide normalization, but it clearly plays a role in the recognition of rotated objects after repeated exposures. The influence of symmetry may be due to increased efficiency of feature extraction on account of feature redundancy (Jolicœur, 1990; Jolicœur \& Humphrey, 1998; Murray et al., 1993). On the other hand, since the viewer need only keep track of the ordering of parts along a single dimension for symmetrical objects, orientation-invariant object representations may have been accessed (McMullen \& Farah, 1991; Tarr \& Pinker, 1990). Given the design of the experiments in this study, it is not possible to distinguish between these two explanations.

Sekuler and Swimmer (2000) demonstrated that increasing the aspect ratio of an object increased the saliency of the axis of elongation. Surprisingly, we found that overall, increasing the saliency of the axis of elongation actually interfered with the identification of rotated objects, since observers took longer to name rotated objects with aspect ratios above 2:1, as compared with those with aspect ratios below $2: 1$. Furthermore, increasing aspect ratios of objects did not change the effects of orientation for any of the object types, suggesting that the null effects on orientation found for the axis of elongation cannot be explained by a lack of salience for elongation.

In summary, the axes of symmetry and elongation did not influence the normalization of rotated objects independently of each other. On first naming, incongruency between the axes of elongation and symmetry increased effects of orientation. After practice in naming the object set, effects of orientation diminished more for objects with congruent axes. As in Experiment 2, the presence of symmetry resulted in a greater reduction in the effects of orientation after repeated naming. Interestingly, increas- ing the aspect ratio of objects interfered with naming, in contrast to the findings of Sekuler and Swimmer (2000).

\section{GENERAL DISCUSSION}

It was predicted that if the axes of elongation and/or symmetry played an important role in the identification of rotated objects when they were first named, interactions between orientation and axis type would occur. An absence of two-way interactions of this type in Experiments 1,2 , and 3 indicated that object axes of elongation and symmetry did not, in isolation, influence effects of orientation during the first block of naming. However, effects of symmetry and elongation together did influence effects of orientation. In Experiment 3, incongruency between the axes defined by symmetry and elongation (wide, symmetrical objects) resulted in increased effects of orientation, particularly at the most extreme orientation tested, $120^{\circ}$. These findings relegate the influence of axes of elongation and symmetry to a relatively minor role in the naming of rotated objects for the first time. As such, they failed to support theories postulating that these axes guide normalization of rotated percepts (or images) to the upright in a bottom-up manner, prior to contacting long-term object representations (Jolicœur, 1990; Marr, 1982; McMullen \& Jolicœur, 1992).

The influence of these axes on effects of orientation did emerge after repeated naming of the same object set. In particular, symmetry diminished effects of orientation after repeated naming, as has been previously reported (McMullen \& Farah, 1991). This effect suggests that the use of the object attribute of symmetry during the process of orientation normalization is learned. Symmetry facilitated naming overall during the first block of naming. However, its relation to orientation normalization was specific to the object set and so emerged only after practice. In particular, congruency between the axes of symmetry and elongation (tall, symmetrical objects) resulted in the smallest effects of orientation with practice. The condition next least affected by orientation was that for wide symmetrical objects. Interestingly, naming for the latter group was most affected by orientation in Block 1 and so showed the greatest diminution in effects of orientation with practice. These effects suggest that incongruency between the two axes increased effects of orientation on first naming but that, with practice, its effect was reduced, perhaps owing to a greater influence of symmetry with practice. The influence of the axis of symmetry on orientation effects after repeated exposure to the objects may have been due to more efficient feature extraction (McMullen \& Farah, 1991) or to access to orientation-invariant object representations for symmetrical objects only (Tarr \& Pinker, 1990).

Finally, it was found that symmetry and elongation influenced each other. In keeping with the conclusions of Gerhardstein and Peterson (1995), effects of elongation were dependent on the presence of an axis of symmetry. 
In the first block of naming and after repeated naming, tall objects benefited most from symmetry cues, as compared with wide objects. In the first block, this was due principally to the incongruency effect found with wide symmetrical objects at $120^{\circ}$, which reduced any advantages for symmetrical wide objects over asymmetrical wide objects. After practice, elongation appeared to have no effect on symmetrical objects. Tall and wide objects were both named quickly and relatively invariant of orientation.

There was only one congruent condition in the present Experiment 3 (tall, symmetrical objects), owing to the availability of such objects. Objects with horizontal axes of symmetry were not included in Experiment 3, owing to the difficulty of finding common objects with this property. If such objects were available and our interpretation of the present data is correct, it is predicted that objects with vertical axes of elongation and horizontal axes of symmetry (incongruent objects) will also show greater effects of orientation on first naming, as compared with other objects. Similarly, objects with horizontal axes of symmetry and elongation (congruent objects) are predicted to show smaller effects of orientation than do other conditions after repeated naming. Tests of these predictions will depend on the ability to find such objects.

The analysis of Block 1 naming times in Experiments 1,2 , and 3 did not support the claim that the axes of elongation and symmetry played a major role in the normalization of line drawings of objects rotated in the plane. It is worth noting, at this point, that presenting the objects as two-dimensional line drawings did not control for all the axis properties inherent in a three-dimensional structure. For example, it is possible that an object could be classed as being tall and symmetrical from one viewpoint but wide and asymmetrical from another (e.g., a truck). However, it seems reasonable to assume that the visual system would be sensitive to cues that aid efficient identification for any given task. If axes of elongation and/or symmetry were important cues for achieving object identification, one would expect the visual system to be sensitive to their presence in a two-dimensional format. In fact, all three experiments demonstrated main effects of elongation and/or symmetry, indicating that these axes may well play a role at some point in the recognition process. However, since cues of elongation and symmetry did not have any major influences on effects of orientation, it appears that these axes were not used substantively to guide normalization of rotated objects when they were first named.

This could mean that there are image or object properties that are more efficient at guiding normalization than are axes of elongation or symmetry. On the other hand, it could be that normalization, in the sense of mental rotation, does not underlie effects of orientation. There are a number of theoretical alternatives accounting for the effects of orientation when rotated objects are named that may prove more viable. For instance, the multiple-views approach (Bülthoff \& Edelman, 1992; Tarr \& Pinker, 1989) proposes that view-specific repre- sentations of objects are stored in memory. The identification of objects seen at unfamiliar viewpoints is dependent on how close in psychological space the unfamiliar viewpoints are to stored familiar viewpoints. Unfamiliar viewpoints are generalized to familiar viewpoints, and the farther away in psychological space an unfamiliar viewpoint is from stored viewpoints, the worse the identification performance. The reduction of orientation effects after repeated naming is explained by the formation of new view-specific representations of the object. However, the multiple-views approach cannot account for differences in orientation effects between symmetrical and asymmetrical objects observed after repeated exposure. Logically, the formation of view-specific representations for asymmetrical objects should be as efficient as those for symmetrical objects.

Alternatively, it has been proposed that long-term object representations are matched to input representations before normalization occurs (Biederman, 1987; Corballis, 1988; De Caro \& Reeves, 2000; Hamm \& McMullen, 1998; Rothwell, 1995). Hamm and McMullen (1998; see also Dickerson \& Humphreys, 1999) demonstrated that when objects were identified at the subordinate level, large effects of orientation were present. However, basicand superordinate-level identification failed to show significant effects of orientation. They concluded that objects are initially identified at a basic level, with subordinate identification relying on further processing of the input representation. This additional processing may include normalization of the input representation.

In accordance with Hamm and McMullen's (1998) argument that normalization occurs after identification, De Caro and Reeves (2000) provided evidence that orientation effects on reaction times reflected a postrecognition process. They briefly presented line drawings of objects at multiple orientations, followed by a mask that was presented at various stimulus onset asynchronies (SOAs). The purpose of the mask was to limit early visual information processing. The SOAs of the mask were varied in order to find the critical SOA required for an optimal level of performance ( $75 \%$ accuracy). The participants' task was to report the orientation of objects and to verify their basic-level names. They found that the critical SOA required to verify the identity of objects was briefer than the critical SOA required to accurately verify orientation. If normalization had preceded identification of the objects, the critical SOA for object verification would have been larger than that for orientation verification. They argued that their results were consistent with a "double-checking" or "rotate-to-orient" hypothesis. The "double-checking" hypothesis purports that orientation effects reflect a normalization process used to verify recognition of a rotated object. The "rotateto-orient" hypothesis purports that orientation effects reflect a normalization process used to determine the orientation of the rotated object.

The lack of major effects of cues of elongation and symmetry on orientation may be explained by these top- 
down approaches, which propose that objects are identified before they are normalized. In this case, the normalization process does not require the extraction of identity-independent stimulus properties, such as principal axes, to guide normalization. In addition, top-down approaches may explain why some of the slopes reported were outside the expected range. The level of categorization at which the objects could be identified was not controlled for in the experiments reported in this paper. It could be that the majority of objects in Experiment 2 and the objects in the wide asymmetrical condition in Experiment 3 (all had slopes above 1,400 deg/sec) were named at the basic level. On the other hand, in line with the "double-checking hypothesis," the particular group of objects showing lesser rates of orientation may have needed little normalization to verify their identity.

In conclusion, this study demonstrated that axes of elongation and/or symmetry did not play a major role in the normalization of rotated images when they were first named, suggesting that they are not good candidates for guiding normalization. However, there was evidence that they were perceptually salient, since in combination, they facilitated or interfered with the identification of rotated objects at the most extreme orientation tested. Their influence during rotated object naming was confined to instances in which the two types of axes defined the same or different orientations and when objects were presented in extreme rotations $\left(120^{\circ}\right)$. When objects were named for the first time, incongruency in the axes defined by elongation and symmetry increased effects of orientation. After repeated naming of the object set, congruency in the axes defined by symmetry and elongation decreased effects of orientation. This effect may have been due, principally, to axes of symmetry, since symmetrical objects always showed a greater reduction in orientation effects after repeated naming, independent of the status of elongation of objects.

Several other investigators have demonstrated that axes of symmetry and elongation influence decisions about the location of these axes. These axes also influence object naming in that symmetrical objects were named more quickly than asymmetrical objects and elongation effects were found that were contingent on symmetry. It is suggested that a failure to find effects of axes on orientation effects in the first block is not due to the fact that these axes are incorrect cues to orientation, but to the fact that the visual system is unable to use orientation cues that form an abstract frame of reference to assist in normalization. This failure has been demonstrated several times (Gauthier \& Tarr, 1997; Gibson \& Peterson, 1994; Ling \& Sanocki, 1995; McMullen et al., 1995; Robertson, Palmer, \& Gomez, 1987). Instead, we support the notion that object identity, at some level, is extracted independently of orientation and that effects of orientation are due to postchecking, which occurs most frequently during subordinate identification. In this view, orientation is derived on the basis of an interaction between the long-term representation of an object and the exemplar image to be named.

\section{REFERENCES}

BIEDERMAN,I. (1987). Recognition-by-components: A theory of human image understanding. Psychological Review, 94, 115-147.

Bülthoff, H. H., \& Edelman, S. (1992). Psychophysical support for a two dimensional view interpolation theory of object recognition. Proceedings of the National Academy of Sciences, 89, 60-64.

Chambers, K. W., McBeath, M. K., Schiano, D. J., \& Metz, E. G. (1999). Tops are more salient than bottoms. Perception \& Psychophysics, 61, 625-635.

CHRISTIE, J. (1999). Image flipper: Experiment presentation software [Computer program]. Dalhousie University, Halifax, Canada. Available at or.psychology.dal.ca/ jc/psycsoft.html.

Corballis, M. C. (1988). Recognition of disoriented shapes. Psychological Review, 95, 115-123.

De Caro, S. A., \& Reeves, A. (2000). Rotating objects to determine orientation, not identity: Evidence from a backward-masking/dualtask procedure. Perception \& Psychophysics, 62, 1356-1366.

Dickerson, J., \& Humphreys, G. W. (1999). On the identification of misoriented objects: Effects of task and level of stimulus description. European Journal of Cognitive Psychology, 11, 145-166.

GAUTHIER, I., \& TARR, M. J. (1997). Orientation priming of novel shapes in the context of viewpoint-dependent recognition. Perception, 26, 51-73.

Gerhardstein,P. C., \& Peterson, M. A. (1995). Object symmetry and axis orientation affect recognition of misoriented objects. Poster session presented at the Annual Meeting of the Association for Research in Vision and Ophthalmology, Fort Lauderdale, FL.

Gibson, B., \& Peterson, M. A. (1994). Does orientation-independent recognition precede orientation-dependent recognition? Evidence from a cuing paradigm. Journal of Experimental Psychology: Human Perception \& Performance, 20, 299-316.

Hamm, J., \& McMullen, P. A. (1998). Effects of orientation on the identification of rotated objects depend on the level of identity. Journal of Experimental Psychology: Human Perception \& Performance, 24, 413-426.

HumphrEYs, G. W., \& RidDOch, M. J. (1984). Routes to object constancy: Implication from neurological impairments of object constancy. Quarterly Journal of Experimental Psychology, 36A, 385-415.

HumphreYs, G. W., \& RidDOCH, M. J. (1985). Authors' correction to "Routes to object constancy." Quarterly Journal of Experimental Psychology, 37A, 493-495.

JoliCGEUR, P. (1985). The time to name disoriented natural objects. Memory \& Cognition, 13, 289-303.

JoLICCUR, P. (1990). Identification of disoriented objects: A dual-systems theory. Mind \& Language, 5, 387-410.

Jolicceur, P., \& Humphrey, K. G. (1998). Perception of rotated twodimensional and three-dimensional objects and visual shapes. In V. Walsh \& J. Kulikowski (Eds.), Perceptual constancy: Why things look as they do (pp. 69-123). New York: Cambridge University Press.

KuČEra, H., \& Francis, W. N. (1967). Computational analysis of present-day American English. Providence, RI: Brown University Press.

Ling, X., \& SANOcKI, T. (1995). Major axes as a moderately abstract model for object recognition. Psychological Science, 6, 370-375.

LiU, T., \& CoOper, L. A. (2001). The influence of task requirements on priming in object decision and matching. Memory \& Cognition, 29, 874-882.

MARR, D. (1982). Vision. San Francisco: Freeman.

Marr, D., \& Nishihara, H. K. (1978). Representation and recognition of the spatial organization of three-dimensional shapes. Proceedings of the Royal Society of London: Series B, 200, 269-294.

McMullen, P. A., \& Farah, M. J. (1991). Viewer-centered and objectcentered representations in the recognition of naturalistic line drawings. Psychological Science, 2, 275-277.

McMullen, P. A., Hamm, J., \& Jolicceur, P. (1995). Rotated object identification with and without orientation cues. Canadian Journal of Experimental Psychology, 49, 133-149.

McMullen, P. A., \& Jolicceur, P. (1990). The spatial frame of reference in object naming and discrimination of left-right reflections. Memory \& Cognition, 18, 99-115. 
McMullen, P. A., \& Jolicceur, P. (1992). The reference frame and effects of orientation on finding the tops of rotated objects. Journal of Experimental Psychology: Human Perception \& Performance, 18, 806-820.

MurRaY,J. E. (1995). The role of attention in the shift from orientationdependent to orientation-invariant identification of disoriented objects. Memory \& Cognition, 23, 49-58.

MurRaY, J. E. (1997). Flipping and spinning: Spatial transformation procedures in the identification of rotated natural objects. Memory \& Cognition, 25, 96-105.

Murray, J. E., Joliceur, P., McMullen, P. A., \& Ingleton, M. (1993). Orientation-invariant transfer of training in the identification of rotated natural objects. Memory \& Cognition, 21, 604-610.

Palmer, S. E. (1990). Modern theories of Gestalt perception. Mind \& Language, 5, 289-323.

Pinker, S. (1984). Visual cognition: An introduction. Cognition, 18, 1-63. Quinlan, P. T., \& Humphreys, G. W. (1993). Perceptual frames of reference and two-dimensional shape recognition: Further examination of internal axes. Perception, 22, 1343-1364.

Riddoch, M. J., \& Humphreys, G. W. (1986). Neurological impairments of object constancy: The effects of orientation and size disparities. Cognitive Neuropsychology, 3, 207-224.

Robertson, L. C., Palmer, S. E., \& Gomez, L. M. (1987). Reference frames in mental rotation. Journal of Experimental Psychology: Human Perception \& Performance, 13, 368-379.

Rothwell, C. A. (1995). Object recognition through invariant indexing. Oxford: Oxford University Press.

Sekuler, A. B. (1996). Axis of elongation can determine reference frames for object perception. Canadian Journal of Experimental Psychology, 50, 270-278.

Sekuler, A. B., \& Swimmer, M. B. (2000). Interactions between symmetry and elongation in determining reference frames for object perception. Canadian Journal of Psychology, 54, 42-55.

Snodgrass, J. G., \& VANDERWART, M. (1980). A standardized set of 260 pictures: Norms for name agreement, image agreement, familiarity, and visual complexity. Journal of Experimental Psychology: Human Learning \& Memory, 6, 174-215.

TARr, M. J., \& Pinker, S. (1989). Mental rotation and orientationdependence in shape recognition. Cognitive Psychology, 5, 233282.

TArr, M. J., \& Pinker, S. (1990). When does human object recognition use a viewer-centered reference frame? Psychological Science, 1, 253-256.

Ullman, S. (1989). Aligning pictorial descriptions: An approach to object recognition. Cognition, 32, 193-254.

VAn Selst, M., \& Jolicceur, P. (1994). A solution to the effect of sample size on outlier elimination. Quarterly Journal of Experimental Psychology, 47A, 631-650.

\section{NOTE}

1. It would have been preferable to include two conditions consisting of tall and wide objects that were symmetrical about the horizontal axis. However, there was not a sufficient number of "real-world" objects that were symmetrical about the horizontal axis and were not symmetrical about the vertical axis.

\section{APPENDIX A \\ List of Tall, Wide, and Non-elongated Objects From Snodgrass and Vanderwart (1980) Used in Experiment 1 and the Mean Elongation Rating for Each Object}

Wide objects:

range $=-0.86$ to -1.0 ; mean rating $=-0.94$.

Non-elongated objects: $\quad$ range $=-0.29$ to 0.29 ; mean rating $=0.0004$.

Tall objects: range $=0.93$ to $1.0 ;$ mean rating $=0.99$.

\begin{tabular}{lllrlr}
\hline \multicolumn{1}{c}{ Wide } & Rating & Non-Elongated & Rating & \multicolumn{1}{c}{ Tall } & Rating \\
\hline sleigh & -1.00 & swan & -0.07 & cat & 1.00 \\
bicycle & -1.00 & deer & 0.07 & seahorse & 1.00 \\
peacock & -0.86 & kettle & 0.29 & gorilla & 1.00 \\
pig & -1.00 & monkey & -0.14 & ostrich & 0.93 \\
airplane & -1.00 & duck & -0.29 & hen & 0.93 \\
train & -1.00 & football helmet & 0.07 & coat & 1.00 \\
frog & -0.86 & goat & 0.06 & harp & 1.00 \\
car & -1.00 & horse & 0.29 & owl & 1.00 \\
lion & -0.93 & dog & 0.00 & well & 1.00 \\
motorcycle & -0.86 & elephant & 0.21 & glass & 1.00 \\
tiger & -1.00 & rabbit & -0.07 & snowman & 1.00 \\
iron & -0.86 & zebra & -0.07 & shirt & 1.00 \\
mouse & -0.93 & ironing board & 0.29 & bottle & 1.00 \\
shoe & -0.86 & donkey & -0.21 & wine glass & 1.00 \\
wagon & -0.93 & cup & -0.14 & dress & 1.00 \\
sofa & -1.00 & piano & -0.07 & doll & 1.00 \\
fry pan & -0.86 & mushroom & 0.21 & lamp & 1.00 \\
accordion & -0.93 & record player & -0.29 & garbage can & 0.93 \\
dresser & -0.93 & ashtray & -0.29 & rocking chair & 1.00 \\
house & -0.93 & tree & 0.29 & pitcher & 0.93 \\
bed & -0.86 & telephone & -0.07 & jacket & 1.00 \\
cannon & -0.93 & basket & -0.14 & candle & 1.00 \\
caterpillar & -1.00 & stool & 0.29 & chair & 1.00 \\
desk & -1.00 & cake & -0.21 & nose & 0.93
\end{tabular}


APPENDIX A (Continued)

\begin{tabular}{|c|c|c|c|c|c|}
\hline Wide & Rating & Non-Elongated & Rating & Tall & Rating \\
\hline \multicolumn{6}{|c|}{ Rated but Not Named } \\
\hline alligator & -1.00 & turtle & -0.86 & camel & 0.79 \\
\hline kangaroo & 0.71 & ant & -0.43 & giraffe & 1.00 \\
\hline leopard & -1.00 & squirrel & 0.79 & cow & -0.71 \\
\hline baby carriage & 0.43 & boot & 0.64 & rooster & 0.79 \\
\hline eagle & 1.00 & snail & -0.43 & grasshopper & -1.00 \\
\hline bear & -1.00 & penguin & 1.00 & fox & -0.50 \\
\hline gun & -0.36 & bus & -1.00 & bowl & -0.79 \\
\hline beetle & -0.79 & rhinoceros & -0.93 & apple & 0.21 \\
\hline watering can & 0.64 & sailboat & 0.50 & oven & 0.71 \\
\hline sheep & -0.93 & fly & -0.36 & swing & 1.00 \\
\hline raccoon & -0.86 & skunk & 0.57 & sweater & 0.57 \\
\hline roller skate & -0.93 & bird & -0.36 & bottle & 0.14 \\
\hline seal & 0.36 & bee & -0.71 & pot & -0.43 \\
\hline helmet & -0.93 & barn & -0.36 & blouse & 0.51 \\
\hline spider & -0.36 & truck & -1.00 & spinning wheel & -0.57 \\
\hline church & 0.57 & helicopter & -0.71 & hat & -0.79 \\
\hline
\end{tabular}

APPENDIX B

List of Symmetrical and Asymmetrical Objects From Snodgrass and Vanderwart (1980) Used in Experiment 2 and the Mean Symmetry Rating for Each Object

Symmetrical objects: $\quad$ range $=1.0-3.2$; mean rating $=2.01$.

Asymmetrical objects: $\quad$ range $=3.4-5.0$; mean rating $=4.45$.

\begin{tabular}{lclc}
\hline Symmetrical & Rating & Asymmetrical & Rating \\
\hline heart & 1.0 & accordion & 3.4 \\
wine glass & 1.2 & eagle & 3.6 \\
water glass & 1.2 & doll & 3.6 \\
wine bottle & 1.3 & fly & 3.7 \\
bell & 1.3 & desk & 3.8 \\
lamp & 1.3 & bed & 3.9 \\
bowl & 1.3 & house & 4.0 \\
envelope & 1.4 & motorcycle & 4.1 \\
salt shaker & 1.5 & frying pan & 4.2 \\
top & 1.5 & penguin & 4.2 \\
sweater & 1.6 & telephone & 4.2 \\
cake & 1.7 & pig & 4.3 \\
crown & 1.7 & hat & 4.3 \\
owl & 1.8 & record player & 4.3 \\
snowman & 1.9 & wagon & 4.5 \\
clown & 1.9 & bus & 4.5 \\
bow & 1.9 & iron & 4.6 \\
strawberry & 1.9 & rooster & 4.6 \\
pear & 2.0 & grasshopper & 4.6 \\
jacket & 2.0 & gorilla & 4.6 \\
butterfly & 2.0 & truck & 4.6 \\
pumpkin & 2.1 & ant & 4.6 \\
pineapple & 2.3 & shoe & 4.7 \\
ashtray & 2.4 & elephant & 4.7 \\
candle & 2.4 & squirrel & 4.8 \\
coat & 2.4 & zebra & 4.8 \\
blouse & 2.4 & piano & 4.8 \\
vest & 2.5 & goat & 4.8 \\
dress & 2.6 & spinning wheel & 4.9 \\
stool & 2.6 & peacock & 4.9 \\
clock & 2.7 & lion & 4.9 \\
umbrella & 2.8 & cow & 4.9 \\
garbage can & 2.8 & boot & 4.9 \\
shirt & 2.9 & barn & 4.9 \\
neck tie & 2.9 & monkey & 4.9 \\
tomato & 3.2 & gun & 5.0 \\
row & & &
\end{tabular}


APPENDIX B (Continued)

\begin{tabular}{|c|c|c|c|c|c|}
\hline Object & Rating & Object & Rating & Object & Rating \\
\hline \multicolumn{6}{|c|}{ Rated but Not Named } \\
\hline drum & 1.0 & ironing board & 3.4 & raccoon & 4.6 \\
\hline nail & 1.0 & basket & 3.5 & deer & 4.6 \\
\hline nut & 1.1 & anchor & 3.5 & chicken & 4.6 \\
\hline barrel & 1.1 & traffic light & 3.5 & hammer & 4.6 \\
\hline light bulb & 1.1 & violin & 3.7 & train & 4.6 \\
\hline guitar & 1.3 & kite & 3.7 & bee & 4.6 \\
\hline ring & 1.4 & well & 3.8 & fox & 4.6 \\
\hline screw & 1.9 & oven & 3.9 & rabbit & 4.6 \\
\hline light switch & 2.1 & sofa & 3.9 & camel & 4.6 \\
\hline lips & 2.0 & bicycle & 4.0 & baby carriage & 4.6 \\
\hline swing & 2.0 & sleigh & 4.1 & leopard & 4.7 \\
\hline leaf & 2.3 & key & 4.1 & seal & 4.7 \\
\hline onion & 2.7 & tree & 4.1 & airplane & 4.7 \\
\hline vase & 2.7 & spider & 4.1 & harp & 4.7 \\
\hline thread & 2.8 & cup & 4.2 & skunk & 4.7 \\
\hline celery & 2.9 & pot & 4.2 & helicopter & 4.7 \\
\hline windmill & 3.0 & kettle & 4.3 & bird & 4.7 \\
\hline padlock & 3.0 & book & 4.4 & nose & 4.7 \\
\hline moon & 3.0 & sheep & 4.4 & rhinoceros & 4.7 \\
\hline chair & 3.1 & mouse & 4.4 & roller skate & 4.8 \\
\hline lemon & 3.1 & rocking chair & 4.4 & foot & 4.8 \\
\hline suitcase & 3.1 & pitcher & 4.4 & ostrich & 4.8 \\
\hline balloon & 3.2 & seahorse & 4.4 & watering can & 4.8 \\
\hline green pepper & 3.2 & tiger & 4.4 & beetle & 4.8 \\
\hline skirt & 3.3 & fox & 4.4 & cat & 4.8 \\
\hline coat hanger & 3.4 & football helmet & 4.5 & bear & 4.8 \\
\hline carrot & 3.4 & frog & 4.5 & kangaroo & 4.9 \\
\hline broom & 3.4 & donkey & 4.5 & swan & 5.0 \\
\hline dresser & 3.4 & alligator & 4.6 & duck & 5.0 \\
\hline
\end{tabular}

APPENDIX C

A List of the Stimuli Presented in Experiment 3 With the Corresponding Correct Responses

\begin{tabular}{|c|c|c|c|c|}
\hline & \multicolumn{2}{|c|}{ Tall Asymmetrical } & \multicolumn{2}{|c|}{ Tall Symmetrical } \\
\hline & Object & Correct Responses & Object & Correct Responses \\
\hline 1 & book & book & anchor & anchor \\
\hline 2 & cat & cat & barrel & barrel \\
\hline 3 & chair & chair & bell & bell \\
\hline 4 & chicken & chicken, hen & candle & candle \\
\hline 5 & eagle & eagle, hawk & coat & coat, jacket, overcoat \\
\hline 6 & ear & ear & dress & dress, housedress \\
\hline 7 & giraffe & giraffe & escalator & escalator, staircase, stairs \\
\hline 8 & gorilla & gorilla, ape & garbage can & garbage can/pail, trash can \\
\hline 9 & jug & jug, pitcher & glass & glass, water glass \\
\hline 10 & ostrich & ostrich & pineapple & pineapple \\
\hline 11 & sock & sock & skirt & skirt \\
\hline 12 & step ladder & step ladder, ladder & window & window \\
\hline 13 & building & building, skyscraper & bottle & bottle, wine bottle \\
\hline 14 & doll & child, kid & lamp & lamp \\
\hline 15 & fire hydrant & fire hydrant & lightbulb & lightbulb \\
\hline 16 & flamingo & flamingo & pants & pants, trousers \\
\hline 17 & flower & flower, tulip & parachute & parachute \\
\hline 18 & harp & harp & pear & pear \\
\hline 19 & hockey stick & hockey stick, stick & rake & rake, hoe \\
\hline 20 & mailbox & letterbox & rocket & rocket, spaceship \\
\hline 21 & nose & nose & saltshaker & saltshaker, shaker \\
\hline 22 & penguin & penguin & tie & tie, necktie \\
\hline 23 & seahorse & seahorse & vase & vase \\
\hline 24 & tree & tree & wine glass & wine glass \\
\hline
\end{tabular}


APPENDIX C (Continued)

\begin{tabular}{|c|c|c|c|c|}
\hline & \multicolumn{2}{|c|}{ Wide Asymmetrical } & \multicolumn{2}{|c|}{ Wide Symmetrical } \\
\hline & Object & Correct Responses & Object & Correct Responses \\
\hline 1 & airplane & airplane, jet & bag & bag, sports bag \\
\hline 2 & bicycle & bicycle, bike & bench & bench, park bench \\
\hline 3 & eye & eye & bow & bow, ribbon, bowtie \\
\hline 4 & fish & fish & bowl & bowl, dish \\
\hline 5 & motorcycle & motorcycle, motorbike & butterfly & butterfly \\
\hline 6 & mouse & mouse, rat & couch & couch, sofa \\
\hline 7 & pig & pig, hog & desk & desk \\
\hline 8 & polar bear & polar bear, bear & dragonfly & dragonfly \\
\hline 9 & rhinoceros & rhinoceros, rhino & envelope & envelope, letter, mail \\
\hline 10 & rollerskate & rollerskate, rollerblade & glasses & glasses, spectacles \\
\hline 11 & tiger & tiger & moth & moth \\
\hline 12 & wagon & wagon, cart, trolley & table & table \\
\hline 13 & alligator & alligator, crocodile & bra & bra \\
\hline 14 & ant & ant & bridge & bridge \\
\hline 15 & bus & bus & brush & brush, scrub brush \\
\hline 16 & caterpillar & caterpillar & canoe & canoe \\
\hline 17 & grasshopper & grasshopper & comb & comb \\
\hline 18 & gun & gun, pistol, revolver & crab & crab \\
\hline 19 & leopard & leopard & fence & fence \\
\hline 20 & seal & seal & hammock & hammock \\
\hline 21 & shoe & shoe & hat & hat \\
\hline 22 & submarine & submarine & lips & lips, mouth \\
\hline 23 & train & train & platter & platter, plate, saucer \\
\hline 24 & truck & truck & watermelon & watermelon, fruit slice \\
\hline
\end{tabular}

(Manuscript received February 26, 2001;

revision accepted for publication May 16, 2002.) 